\title{
Clinical, MRI and Arthroscopic Correlation in Internal Derangement of Knee
}

\author{
Sharma UK, ${ }^{1}$ Shrestha BK, ${ }^{2}$ Rijal S, ${ }^{2}$ Bijukachhe $B,{ }^{2}$ Barakoti ${ }^{2},{ }^{2}$ Banskota $B,{ }^{2}$ Pradhan $I^{2}$ \\ Banskota $\mathrm{AK}^{2}$
}

${ }^{1}$ Department of Radiology

${ }^{2}$ Department of Orthopaedics

B\&B Teaching Hospital

Lalitpur, Nepal.

\section{Corresponding Author}

Umesh Kumar Sharma

Department of Radiology

B\&B Teaching Hospital

Gwarko, Lalitpur, Nepal.

Email: druksharma@hotmail.com

Citation

Sharma UK, Shrestha BK, Rijal S,Bijukachhe B, Barakoti R, Banskota B, Pradhan I, Banskota AK. Clinical, MRI and arthoscopic correlation in internal derrangement of knee. Kathmandu Univ Med J 2011;35(3):174-8.

\begin{abstract}
Background

The traumatic or degenerative internal derangement of the knee requires certain investigations for the establishment of diagnosis, in addition to clinical history and a thorough physical examination. The use of arthrography and arthroscopy improves the accuracy of the diagnosis. MRI scanning of the knee joint has often been regarded as the noninvasive alternative to diagnostic arthroscopy.

\section{Objective}

The purpose of the study was to correlate clinical and low field MRI findings with arthroscopy in internal derangement of the knee.

Methods

Forty one patients with suspected internal derangement of the knee were subjected to MR examination followed by arthroscopy. Clinical criteria used were history, mode of injury, Mc Murray's, Apley's grinding, Thessaly's test for meniscal injury. Drawer test was considered to be essential for clinical diagnosis of cruciate ligament injury. MRI of the knee was performed in low field open magnet (0.35T, Magnetom C, Seimens). Arthroscopy was done within two months of MR examination and was considered gold standard for the internal derangement of the knee.
\end{abstract}

\section{Results}

The sensitivity, specificity, diagnostic accuracy of clinical examination were $96.1 \%$, $33.3 \%$ and $73.1 \%$ respectively for medial meniscal tear; $38.4 \%, 96.4 \%$ and $78.1 \%$ respectively for lateral meniscal tear. The sensitivity, specificity, diagnostic accuracy of MRI were $92.3 \%, 100 \%$ and $95.1 \%$ for medial meniscal tear; $84.6 \% 96.4 \%$ and $92.6 \%$ respectively for lateral meniscal tear.

\section{Conclusion}

Clinical examination showed higher sensitivity for medial meniscal tear compared to MRI, however with low specificity and diagnostic accuracy. Low field MRI showed high sensitivity, specificity, diagnostic accuracy for meniscal and cruciate ligament injury, in addition to associated derangement like articular cartilage damage, synovial thickening.

\section{KEY WORDS}

arthroscospy, knee, MRI.

\section{INTRODUCTION}

The traumatic or degenerative internal derangement of the knee is a common entity and may require certain studies for the establishment of diagnosis, in addition to clinical history and a thorough physical examination. The use of arthrography and arthroscopy improves the accuracy of the diagnosis, both are invasive and can cause complications. The reported accuracy of arthrography has ranged widely from 67 to $97 \%$, and the technique requires a person who is skilled in reporting and interpreting the results. ${ }^{1-3}$
It also involves exposure to ionizing radiation. Diagnostic arthroscopy is an important advance, improving diagnostic accuracy 64 to 94 per cent. ${ }^{2,4-6}$ However it is an invasive procedure, with the possible attendant complications of infection, hemarthrosis, adhesions, reflex sympathetic dystrophy.

MRI scanning of the knee joint has often been regarded as the noninvasive alternative to diagnostic arthroscopy. MRI 


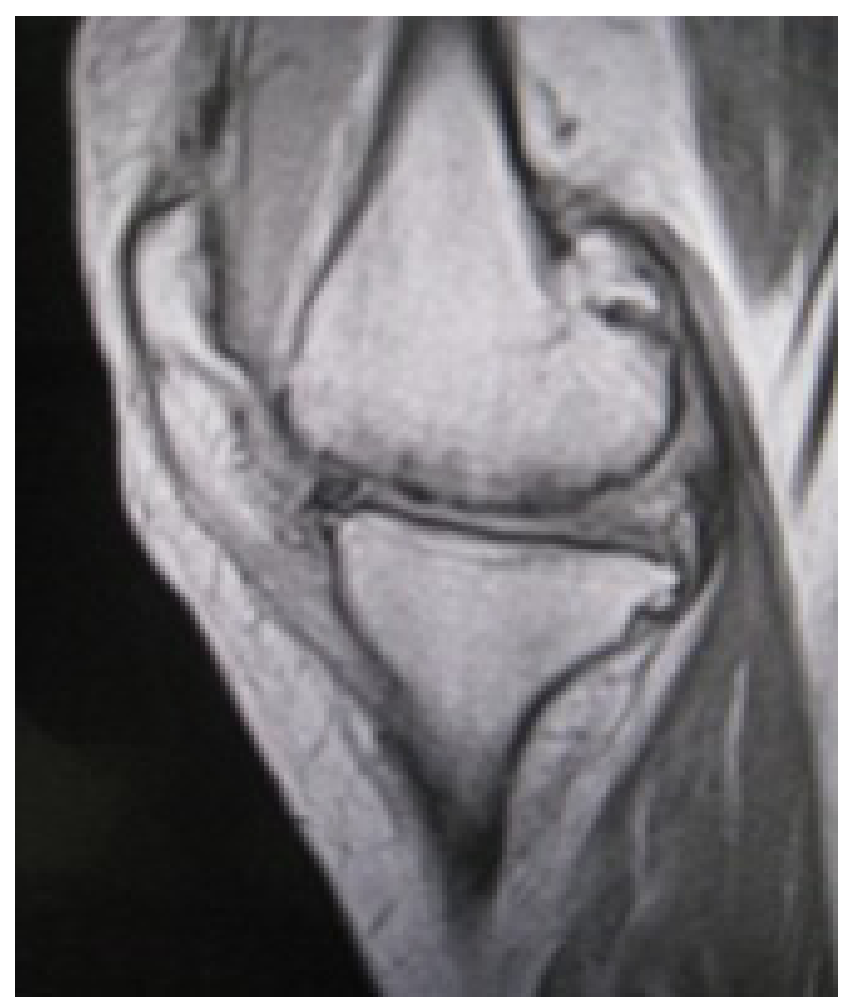

Figure 1. Absence of medial meniscus. There is hyperintense signal intensity on PD sagittal image in the area that was previously occupied by the medial meniscus. Associated articular cartilage loss seen in the femoral condyle with signal change in the subchondral marrow.

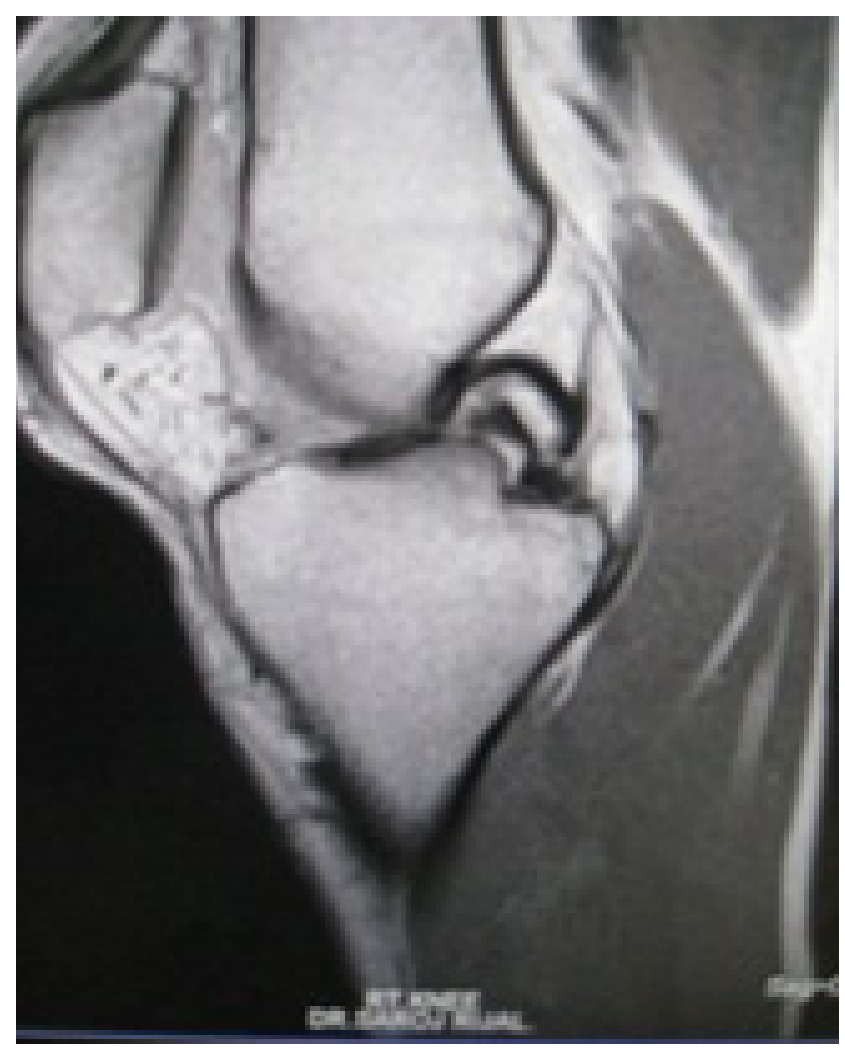

Figure 2. Double PCL sign in bucket handle medial meniscal tear.

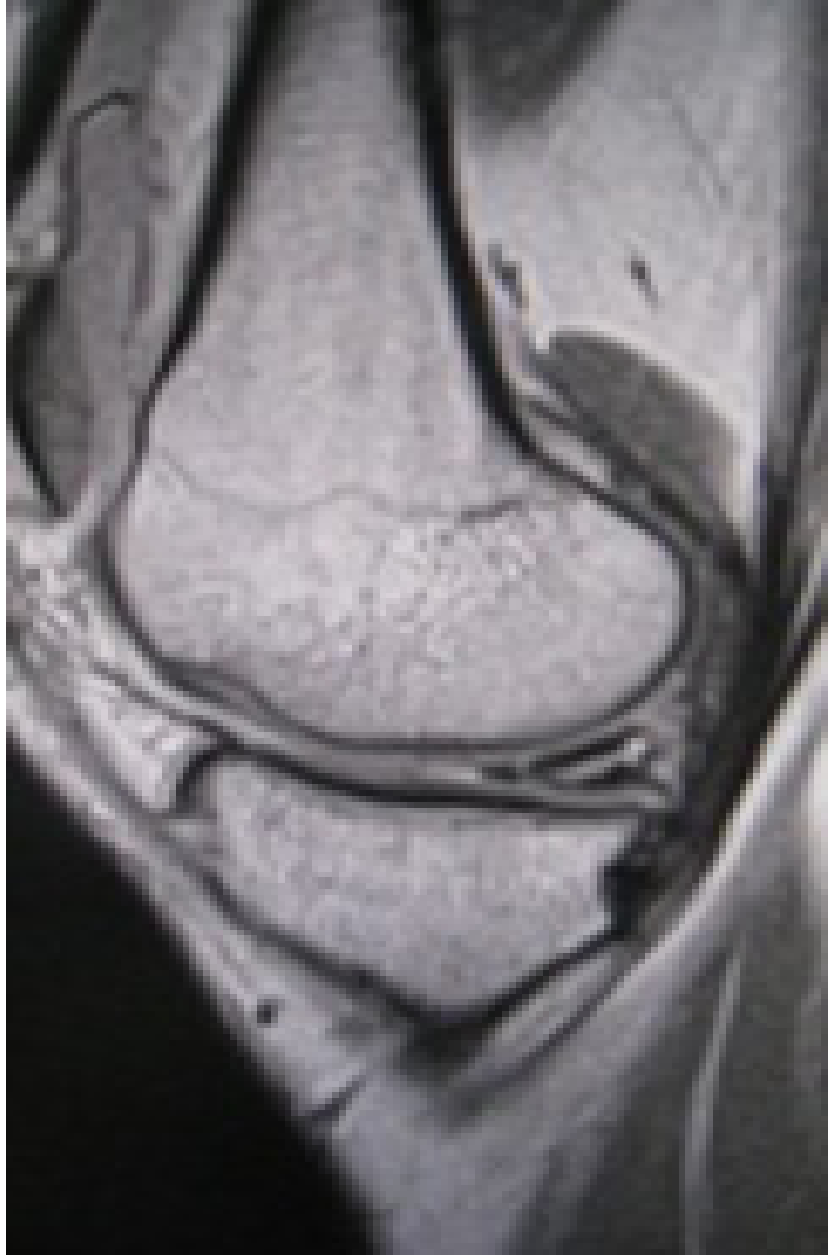

Figure 3. Sagittal PD image showing horizontal tear in the posterior horn of medial meniscus.

scan is routinely used to support the diagnosis for meniscal or cruciate ligament injuries prior to recommending arthroscopic examination and surgery. Identification of meniscal tears can be difficult to interpret and can be observer dependant as well as dependent upon the sensitivity of the scanner. Similar difficulties may exist in clinical examination as well. Our objective was to compare and correlate clinical, MRI, and arthroscopic findings in the diagnosis of meniscal and cruciate ligament injuries.

\section{METHODS}

Forty one patients with suspected internal derangement of the knee were subjected to MR examination and followed by arthroscopy. Clinical criteria used were history, mode of injury, Mc Murray's, Apleys grinding, Thessaly's test for meniscal injury. Drawer test was considered to be essential for clinical diagnosis of cruciate ligament injury.

MR examination of the knee was performed in a low field open 0.35T magnet (Magnetom C, Siemens). The normal slice thickness was $4 \mathrm{~mm}$. PD sagittal, T2 weighted axial and sagittal, T1 weighted and STIR coronal images were obtained. The criterion that was used for determining the presence of a meniscal tear was the presence of a high signal that extended one of the borders of the meniscus, 


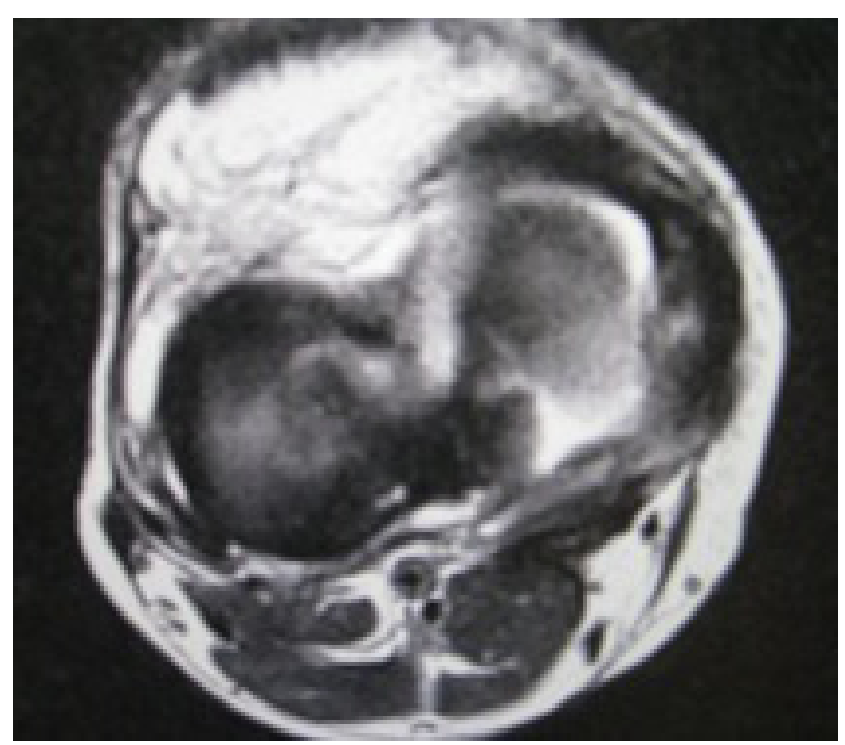

Figure 4. T2 axial image of the knee. Wide tear seen in the posterior horn of medial meniscus.

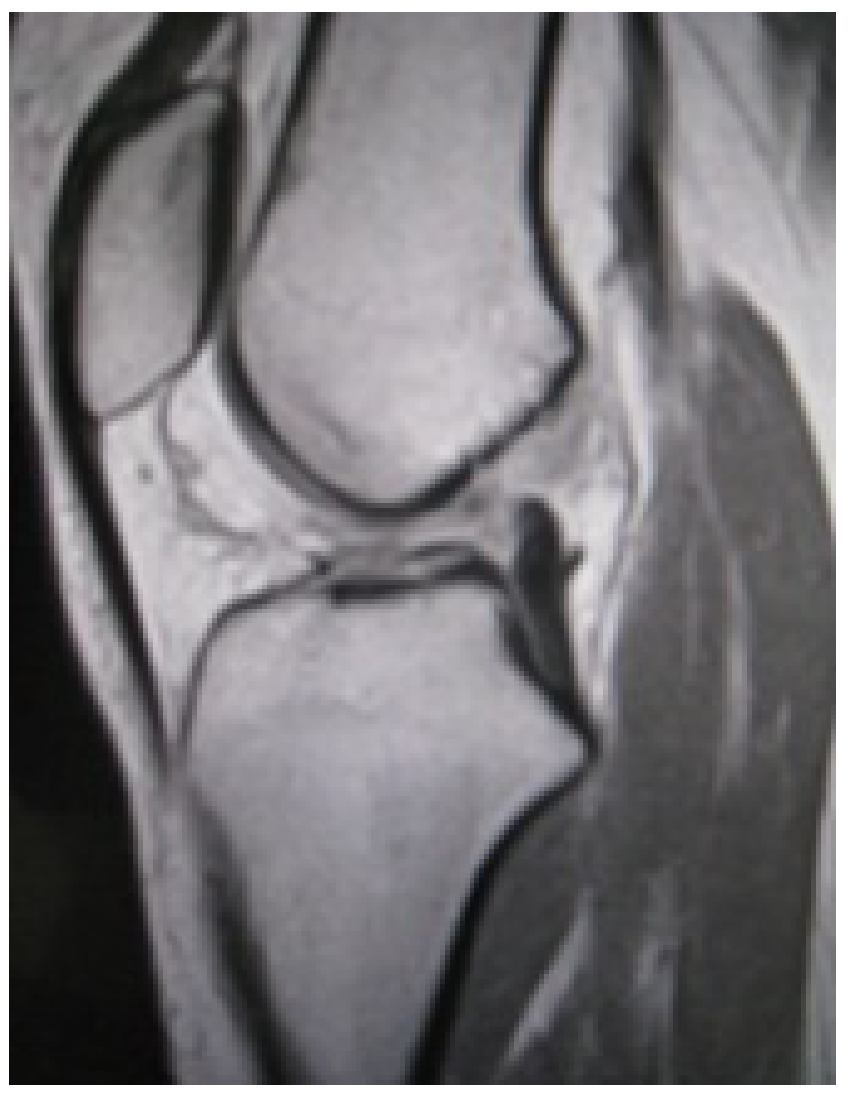

Figure 5. T2 sagittal image: Complete tear of ACL.

different types of meniscal tear (like bucket handle, flap, radial). The cruciate ligament was described as either well visualized, intact, not well visualized, or partial tear. MR examination of knee was performed before arthroscopy was done. The time interval between the performance of the scan and the arthroscopy was two months.

Arthroscopic examination were carried out through the use of two inferior parapatellar portal under general/or spinal anesthesia. A routine sequence that included evaluation of the patellofemoral joint, examination of the intercondylar notch. The medial and lateral compartments were evaluated for osseus, cruciate and meniscal abnormalities. Record of clinical, MRI and arthroscopic findings were kept and compared. Arthroscopic findings were regarded as the gold standard.

The statistical analysis was done by using SPSS. Sensitivity, specificity, positive predictive value, negative predictive value were calculated.

\section{RESULTS}

Of the total 41 patients, 19 were male and 22 female. The mode of injury was sport in 12 , where as non sport in 29. The age ranged from 18 to 63 years. Right knee was affected in 23 and left knee in 18 patients.

There were 35 patients clinically suspected to have medial meniscal tear, out of which arthroscopy confirmed only in 25 patients with sensitivity of $96.1 \%, 33.3 \%$ specificity and $73.1 \%$ diagnostic accuracy. The positive and negative predictive value were $71.4 \%$ and $83.3 \%$ respectively.

Arthrocopy showed lateral meniscal tear in 5 of 6 clinically suspected patients, where as in 8 patients arthroscopy showed tear in 35 clinically negative for lateral meniscal tear with $38.4 \%$ sensitivity, $96.4 \%$ specificity and $78.0 \%$ diagnostic accuracy. The positive predictive value was $83.3 \%$ and negative predictive value was $77.1 \%$. Two patients had discoid lateral meniscus tear.

MRI showed medial meniscal tear in 24 patients, which were confirmed by arthroscopy, where as MRI didn't show tear in two patients with sensitivity, specificity and diagnostic accuracy of $92.3 \%, 100 \%$ and $95.1 \%$ respectively. The positive and negative predictive value for medial meniscal tear by MRI were $100 \%$ and $88.2 \%$ respectively.

Arthroscopy confirmed lateral meniscal tear in 11 of 12 patients suspected in MRI with 2 false negative results. The sensitivity, specificity and diagnostic accuracy were $84.6 \%$, $96.4 \%$ and $92.6 \%$ respectively. The positive and negative predictive value for lateral meniscal tear were $91.6 \%$ and $93.1 \%$ respectively.

In two patients $A C L$ tear were suspected clinically, which were confirmed both by MRI and arthroscopy. Combined medial and lateral meniscus tear were seen in four cases. Chondral damage were shown in 9 patients by MRI which were confirmed by arthroscopy.

\section{DISCUSSION}

MRI of the knee is highly sensitive and specific for the detection of internal derangement of knee. In our study with low field MRI showed better sensitivity and specificity compared to clinical examination in the detection of meniscal tears, which was proved in arthroscopic examination. Discoid lateral meniscus was found in two cases, one in young female and another middle age male. 
The mode of injury in these two cases were non-sport. The type of tear were horizontal involving the surface, where as in medial meniscus tear the types of tear were various. Although the number of cases of ACL tear is few, clinical and MRI had shown $100 \%$ accuracy in the detection of the tear. There was additional advantage of MRI in the detection of chondral damage, synovial thickening etc. The patient age group was heterogeneous with predominant middle age group from 35 to 50 years. In non-sport injury the tear of the meniscus was often degenerative. Associated chondral damage was found in nine patients of degenerative meniscal injury. The study showed generally more painful knees in patients with chondral loss than without chondral damage. The study population is small, however it showed satisfactory diagnostic accuracy comparable to the figures in other studies of high field MRI. The limitation was longer examination time in low field MRI.

Rayan F et al evaluated 131 patients of traumatic meniscal and anterior cruciate ligament injury. The study showed clinical examination had better sensitivity (0.86 vs 0.76 ), specificity ( 0.73 vs 0.52 ), predictive values, and diagnostic accuracy in comparison to MRI scan in diagnosing of medial meniscal tears. These parameters showed marginal difference in lateral meniscal and anterior cruciate ligament injuries with sensitivity ( 0.56 vs 0.61 ), specificity ( 0.95 vs 0.92 ) for LMT and sensitivity (0.77 vs 0.81$)$, specificity (1.0 vs 0.96) for ACL injury. ${ }^{7}$

In the study done by Chang CY et al in 148 patients, they found $92 \%$ for sensitivity and $87 \%$ for specificity for meniscal tears. ${ }^{8}$ Munshi et al studied 23 patients of hemarthrosis who had MRI scans followed by arthroscopy. Higher sensitivity and the conclusion was made that prospective use of MRI could have prevented $22 \%$ of diagnostic arthroscopic procedures. ${ }^{9}$

Lundberg $M$ et al found sensitivity and specificity of $74 \%$ and $66 \%$, respectively, for medial and $50 \%$ and $84 \%$ for lateral meniscus. ${ }^{10}$ Barronian $\mathrm{AD}$ et al found $\mathrm{MRI}$ to be $100 \%$ sensitive for medial meniscal tears and $73 \%$ for lateral meniscal tears. ${ }^{11}$

Polly DW et al in comparative study in 54 patients with selective MRI and arthroscopy found the sensitivity, specificity and accuracy of selective MRI 95.8\%, 100\% and $98 \%$ respectively for medial meniscal tear, $66.7 \%, 95.1 \%$ and $90 \%$ for lateral meniscal tear, $100 \%, 96.9 \%$ and $97.3 \%$ for ACL tears. ${ }^{6}$

Rangger Cet al studied 121 patients and concluded that MRI should be an essential diagnostic tool before arthroscopy. ${ }^{12}$ In a retrospective study by Mohan BR et al in 130 patients, diagnostic accuracy of clinical examination was $88 \%$ for medial meniscal tears and $92 \%$ for lateral meniscal tears; they concluded that clinical diagnosis of meniscal tears is as reliable as the magnetic resonance imaging scan. ${ }^{13}$

Vaz CE et al performed MRI evaluation in identifying traumatic intraarticular knee lesions. They found $97.5 \%$ sensitivity, $92.9 \%$ specificity, $93.9 \%$ positive predictive value for medial meniscal lesions; 91.9\%sensitivity, 93.6\%specificity, $92.7 \%$ positive predictive value for lateral meniscal lesions. They concluded MRI as a satisfactory diagnostic tool for evaluating meniscal and ligamentous lesions of the knee, but it is unable to clearly identify articular cartilage lesions. ${ }^{14}$

Thomas $\mathrm{S}$ et al concluded lower accuracy of MRI in detecting $\mathrm{ACl}$ and meniscal pathology. The overall accuracy for MRI was 91,68 and $86 \%$ for detecting ACL, medial and lateral meniscal tears respectively. ${ }^{15}$

Theofilos $\mathrm{K}$ et al found the clinical Thessaly test at 20 degree of flexion had a high diagnostic accuracy rate of $94 \%$ in the detection of tears of the medial meniscus and $96 \%$ in the detection of tears of the lateral meniscus. ${ }^{16}$

\section{CONCLUSIONS}

Medial meniscal tear is more common than lateral meniscal tear. Clinical examination has high sensitivity and low specificity for the detection of medial meniscal tear compared to that of MRI. MRI is superior in the detection of lateral meniscal tear. The low field MRI showed better diagnostic accuracy for the detection of meniscal tear, in addition to other associated derangements like articular cartilage loss, synovial thickening, which was comparable to the figures found in other studies.

\section{REFERENCES}

1. Freiberger RH, Killoran PJ and Cardona Gonzalo: Arthrography of the knee by double contrast method. Am J Roentgenol 1966; 97:736-47.

2. Gillies, Hamish and Seligson, David:Precision in the diagnosis of meniscal lesions: a comparison of clinical evaluation, Arthrography and arthroscopy. J Bone and Joint Surg 1979; 61-A:343-6.

3. Hall FM: Pitfalls in the assessment of the mnisci by knee arthrography. Radiol Clin Noth America 1981; 19:305-28.

4. De Haven, K.E. and Collins HR: Diagnosis of internal derangement of the knee. The role of Arthrography. J Bone and Joint Surg 1975; 57A:802-10

5. Ireland John, Trickey EL and Stroker DJ: Arthroscopy and Arhrography of the knee. A critical review. J Bone and Joint Surg 1980; 62-B(1):3-6.

6. DW Polly, JJ Collaghan, RA Sikes, JM McCabe, K McMahon, CG Savory. The accuracy of selective magnetic resonance imaging compared with the findings of arthroscopy of the knee. J Bone Joint Surg 1988;70:192-8.

7. Rayan F, Bhonsle S, Shukla DD. Clinical, MRI, and arthroscopic correlation in meniscal and anterior cruciate ligament injuries. Int Orthop 2009; 33(1):129-32.

8. Chang $\mathrm{CY}, \mathrm{Wu} \mathrm{HT}$, Huang $\mathrm{TF}$, Ma HL, Hung SC. Imaging evaluation of meniscal injury of the knee joint: a comparative MR imaging and arthroscopic study. Clin Imaging 2004;28(5):372-6.

9. Munshi M, Davidson M, MacDonald PB, Froese W, Sutherland K. The efficacy of magnetic resonance imaging in acute knee injuries. Clin J Sport Med 2000;10(1):34-9. 
10. Lundberg $M$, Odensten $M$, Thumas KA, Messner K. The diagnostic validity of magnetic resonance imaging in acute knee injuries with hemarthrosis. A single-blinded evaluation in 69 patients using highfield MRI before arthroscopy. Int J Sports Med 1996;17(3):218-22.

11. Barronian AD, Zoltan JD, Bucon KA. Magnetic resonance imaging of the knee:correlation with arthroscopy. Arthroscopy 1989;5(3):18791.

12. Rangger C, Klestil T, Kanthrein A, Inderster A, Hamid L. Influence of magnetic resonance imaging on indications for arthroscopy of the knee. Clin Othop Relat Res 1996;330:133-42.

13. Mohan BR, Gosal HS. Reliability of clinical diagnosis in meniscal tears. Int Orthop 2007;31(1):57-60.
14. Vaz CE, Camargo OP, Santana PJ, Valezi AC. Acuracy of magnetic resonance in identifying traumatic intraarticular lesion. Clinics (Sao Paulo) 2005 Dec;60(6):445-50.

15. Thomas S, Pullaqura M, Robinson E, Cohen A, Banaskiewicz P. The value of magnetic resonance imaging in our current management of $\mathrm{ACL}$ and meniscal injuries. Knee Surg Sports traumatol Athrosc 2007;15(5):533-6.

16. Theofiolos Karachalios, Michael Hantes, Aristides H. Zibis, Vasilios Zachos, Apostolos H. Karantanas N. Malizos. Diagnostic accuracy of a new clinical test (the Thessaly test) for early detection of meniscal tears. J Bone Joint Surg 2005;87:955-62. 